\title{
The Accuracy of Non-Conventional Methods Compared to Conventional Methods for Diagnosis of Bloodstream Infections
}

\author{
Raghdah A Abdulmajeed ${ }^{1}$, Asif Jiman-Fatani ${ }^{2,4}$ and Dalia EL-Hossary ${ }^{3 *}$ \\ ${ }^{1}$ Regional lab Makkah, Ministry of Health, Makkah, Kingdom of Saudi Arabia \\ ${ }^{2}$ Department of Medical Microbiology and Parasitological, Faculty of Medicine, \\ King Abdulaziz University, Jeddah, Kingdom of Saudi Arabia \\ ${ }^{3}$ Department of Medical Microbiology and Immunology, Faculty of Medicine, \\ Zagazig University, Egypt \\ ${ }^{4}$ Clinical and Molecular Microbiology Laboratory, King Abdulaziz University \\ Hospital, Jeddah, Kingdom of Saudi Arabia \\ *Corresponding Author: Dalia EL-Hossary, Department of Medical Microbiology \\ and Immunology, Faculty of Medicine, Zagazig University, Egypt.
}

Received: July 19, 2021

Published: August 12, 2021

(C) All rights are reserved by Dalia EL

-Hossary., et al.

\begin{abstract}
Bloodstream infections (BSI) are severe infections leading to potentially life-threatening illnesses that requires quick diagnosis, which ensures that the patient is administered appropriate antimicrobials and proper care. The identification of microorganisms from positive blood cultures by a conventional method usually takes several days. In this study, we investigated the performance of three rapid technologies viz. MALDI-TOF, FilmArray (BioFire), and Nanosphere for their ability to identify the microorganisms present in positive blood culture bottles either directly, before subculture, or after subculture and compare it with VITEK 2 method (gold standard). A total of 1,766 microorganisms were isolated in this study. MALDI-TOF identified 437 Gram-positive bacteria (out of 443 ) Gram-positive isolates from a colony subculture, and showed sensitivity, Positive predictive value (PPV), and accuracy of 98.7\%, 99\%, and 98\%, respectively. MALDI-TOF correctly identified 403 Gram-negative bacteria (out of 407) with 99\% sensitivity, PPV was 100\%, and 99\% accuracy. Nanosphere BC-GN assay accurately identified 343 Gram-negative bacteria out of 407 Gram-negative bacteria with sensitivity, PPV, and accuracy of $84.3 \%, 99 \%$, and 83\%, respectively. In our study, out of 443 Gram-positive bacteria, 435 were identified by BioFire with sensitivity, PPV, and accuracy of $98.2 \%, 99 \%$, and $98.2 \%$, respectively. BioFire could also identify 59 yeast isolates out of 66 with 89\% sensitivity, 100\% PPV, and 89\% accuracy. In conclusion, these non-conventional technologies provided fast and reliable results. Adopting of these technologies should be encouraged in the microbiology labs of the hospitals.
\end{abstract}

Keywords: Bloodstream Infection; VITEK 2; MALDI-TOF; BioFire; Nanosphere

\section{Abbreviations}

BSI: Bloodstream Infection; PPV: Positive Predictive Value; AST: Antimicrobial-susceptibility Testing; FA BCID: FilmArray Blood
Culture Identification; PCR: Polymerase Chain Reaction; KPC: Carbapenem-Resistance Gene; mecA: Methicillin-resistance Gene; Vana/B: Vancomycin-resistance Gene; BC-GP: The Verigene Blood

Citation: Dalia EL-Hossary., et al. "The Accuracy of Non-Conventional Methods Compared to Conventional Methods for Diagnosis of Bloodstream Infections". Acta Scientific Microbiology 4.9 (2021): 69-80. 
Culture Gram-positive; BC-GN: The Verigene Blood Culture Gramnegative; ESBLs: Extended-spectrum ß-lactamases; MALDI-TOF MS: Matrix-assisted Laser Desorption Ionization Time-of-Flight Mass Spectrometry; KAUH: King Abdulaziz University Hospital; SDA: Sabouraud Dextrose Agar; QC: Quality Control; CHCA: $\alpha$-cyano-4-hydroxycinnamic Acid

\section{Introduction}

Bloodstream infections (BSI) are considered one of the most severe infections that cause potentially life-threatening illnesses [1]. Bloodstream infections also lead to significant morbidity and mortality [2], which drain out the finances of the healthcare system [3]. Therefore, it is vital to identify the causative microorganisms in the BSI cases as soon as possible to enable appropriate selection of the antimicrobials and medical care thereby, reducing the risk of morbidity and mortality [4].

Automation with continuous monitoring of the liquid blood cultures is currently the gold standard for detecting causative organisms in BSIs [5]. The positive blood culture bottles are subcultured on solid media. The initial identification is performed by a Gram stain test, followed by phenotypic determination and antimicrobial-susceptibility testing (AST) [5]. This standard liquid blood culture diagnostic method, which requires up to five days, is a timeconsuming process that forces clinicians to treat their patients empirically with antimicrobial agents [6]. Therefore, to improve patients' outcomes, it is vital to identify the causative microorganism as early as possible and determine its antimicrobial-susceptibility pattern [7]. Early identification of the bacteria and detection of antimicrobial resistance genes directly through molecular assays can significantly improve the final outcome of the patient [8]. It assists clinicians in choosing the most appropriate antimicrobial therapy for patients with BSI [9].

The FilmArray blood culture identification (FA BCID) (BioFire) panel is a molecular method, with an automated, nested multiplex polymerase chain reaction (PCR) assay that identifies common microorganisms directly from positive blood culture bottles. It is an easy and rapid test that requires about 2 minutes to process the sample, and rapid processing inside the instrument giving the results within an hour [7]. This method is designed to identify 24 microorganisms that commonly cause sepsis. The panel includes 8 Gram-positive (GP) organisms, including Staphylococcus spp., Staphylococcus aureus, Listeria monocytogenes, Streptococcus spp., Streptococcus agalactiae, Streptococcus pyogenes, Streptococcus pneumoniae, and Enterococcus. Also, 11 Gram-negative (GN) organisms included in the panel are Haemophilus influenzae, Neisseria meningitides, Enterobacteriaceae, Enterobacter cloacae complex, Escherichia coli, Klebsiella oxytoca, Klebsiella pneumoniae, Proteus, Serratia marcescens, Pseudomonas aeruginosa, and Acinetobacter baumannii; and 5 Candida species, including C. albicans, C. glabrata, C. krusei, C. parapsilosis, and C. tropicalis. It can also identify three antimicrobial-resistance genes: carbapenem-resistance KPC gene, methicillin-resistance mecA gene, and vancomycin-resistance vanA/B gene $[10,11]$.

The second molecular method used is Verigene (Nanosphere), which is an automated 2.5-hour microarray test that can detect significant Gram-negative and Gram-positive organisms in two separate panels directly from positive blood cultures, with only 10 minutes of preparation time $[12,13]$. The Verigene blood culture Gram-positive (BC-GP) assay examines four genera (Staphylococcus, Streptococcus, Micrococcus, and Listeria), nine species (Staphylococcus aureus, Staphylococcus lugdunensis, Staphylococcus epidermidis, Streptococcus anginosus group, Streptococcus agalactiae, Streptococcus pneumoniae, Streptococcus pyogenes, Enterococcus faecium, and Enterococcus faecalis), and three resistance markers, the mecA, vanA, and vanB genes [13,14]. The Verigene blood culture Gram-negative (BC-GN) assay detects five species (Klebsiella pneumoniae, Klebsiella oxytoca, Escherichia coli, Serratia marcescens, and Pseudomonas aeruginosa) and four genera (Citrobacter spp., Proteus spp., Acinetobacter spp., and Enterobacter spp.), plus six resistance markers, including the carbapenem resistance genes (KPC, VIM, NDM, IMP, and OXA) and the extended-spectrum ß-lactamases (ESBLs) gene (CTX-M) [15].

Matrix-assisted laser desorption ionization time-of-flight mass spectrometry (MALDI-TOF MS) is another modern technology that is frequently used for the rapid identification of organisms by recognizing the protein fingerprints of different microorganisms [16]. MALDI-TOF is an automated bacterial and fungal identification system used effectively to identify bacterial and fungal colonies subcultured from positive blood culture bottles $[17,18]$. Furthermore, other studies have also demonstrated the use of MALDI-TOF for bacterial identification directly from positive blood culture bottles [19]. MALDI-TOF MS requires a small sample volume and inexpensive reagents to yield results in a rapid turn-around time (10 minutes) [20]. MALDI-TOF relies on protein analysis and characterization to identify microorganisms [21]. 


\section{Aim of the Study}

The present study aims to compare the non conventional rapid technologies for identifying microorganisms versus the conventional methods. Also, we have evaluated the advantages and disadvantages of using these rapid methods for BSI microbiological diagnosis.

\section{Materials and Methods}

Study design and setting

This study was conducted in the Clinical and Molecular Microbiology Laboratory at King Abdulaziz University Hospital (KAUH), Jeddah. All included positive blood cultures in this study were obtained from the patients admitted to KAUH from June 2017 to June 2020. Repetitive blood culture samples from the same patient were excluded. We evaluated the performance of the FilmArray (BioFire), Verigene (Nanosphere), and VITEK MS (MALDI-TOF) bacterial identification methods with the VITEK 2 system, which is the gold standard method for the identification of Gram-positive and Gram-negative bacteria and yeast. We evaluated BioFire to identify Gram-positive bacteria and yeast, Nanosphere for identifying Gram-negative bacteria, and MALDI-TOF to identify both Grampositive and Gram-negative bacteria. A total of 1,766 blood culture isolates were analyzed in the study.

\section{Ethical consideration}

The study was approved by the Research Ethics Committee, Unit of Biomedical Ethics, Faculty of Medicine, King Abdulaziz University (Reference Number: 223-14).

\section{Method}

- Blood culture bottles BacT/ALERT SA Standard Aerobic (blue tap) and BacT/ALERT SN Standard Anaerobic (purple tap) were used in this study. Each bottle was inoculated with $5 \mathrm{ml}$ of the patient's blood sample. The culture bottles were further incubated in BacT/ALERT 3D (BioMérieux, Durham, NC, USA) till a positive signal was observed after a maximum incubation of 5 days $[22,23]$.

- Positive blood culture bottles were processed inside a class II safety cabinet for Gram staining. Simultaneously, the samples were cultured on Blood agar, Chocolate agar, and MacConkey agar for bacteria, while Sabouraud dextrose agar (SDA) was used to culture yeast (Saudi Prepared Media Laboratories, Riyadh, KSA). The MacConkey agar and SDA containing culture plates were incubated for $18-24$ hours at $35-37^{\circ} \mathrm{C}$ in an ordinary incubator (Forma Scientific Incubator, Germany). Blood agar and Chocolate agar plates were incubated at 35 $37^{\circ} \mathrm{C}$ in a $5-10 \% \mathrm{CO}_{2}$ incubator (Sanyo $\mathrm{CO}_{2}$ Incubator, Japan).

- The Gram staining results assisted in selecting the method (Nanosphere or BioFire) to be used for direct identification of bacteria from positive blood culture bottles.

- All the colonies from the subcultured plates were identified by Gram staining for further identification by VITEK 2 system. However, colonies of 850 isolates were identified by MALDI-TOF before identification by VITEK 2.

\section{VITEK 2 system (BioMérieux, Marcy l'Etoile, France)}

The identifying cards were chosen according to the Gram staining results and biochemical tests. Subsequently, a suspension was prepared from a pure subculture plate by mixing a colony with 3.0 $\mathrm{ml}$ of $0.45 \%$ sterile saline, which was aseptically added into a clear plastic test tube. The density of each suspension was adjusted to the McFarland standard of 0.5 to 0.63 using the VITEK 2 DensiCheck densitometer $[24,25]$. The suspension tube was placed in the cassette, followed by an empty tube. The VITEK 2 ID card was then inserted into the suspension tube [26]. The VITEK 2 system also identified the yeast isolates. The isolated pure colonies were selected from the SDA plates, and purity plating was done to ensure that a pure culture was used for testing. A sterile loop transferred a sufficient number of morphologically similar colonies to the saline tube, and the density of the suspension was checked using VITEK 2 DensiCheck, which was equivalent to McFarland standard of 2.0. Subsequently, the suspension tube was placed in the cassette, followed by an empty tube, and the identification card for yeast was placed in the suspension tube [27]. The AST card was placed next to the VITEK 2 card into the empty tube. Finally, cassettes were loaded into the VITEK 2 system. When the sample cycle was finished, the cassettes and the tubes were discarded. Following the sample cycle of 10-15 h for Gram-negative, 16-24 h for Gram-positive, 24 - 48h for yeast, the results were ready [26,27].

\section{VITEK MS (MALDI-TOF) (BioMérieux, Marcy l'Etoile, France)}

A thin layer was made using a $1 \mu \mathrm{L}$ loop from a colony (from a subculture plate) to a "spot" on the VITEK MS-DS target slide, and $1 \mu \mathrm{l}$ of the $\alpha$-cyano-4-hydroxycinnamic acid (CHCA) VITEK MS matrix solution was mixed with it [20]. A quality control (QC) check was conducted with each run, which was positive for E. faecalis ATCC 19433 and negative for the CHCA VITEK MS matrix solution alone (without the organism). Also, E. coli ATCC 8939 calibration 
was performed by adding a $1 \mu \mathrm{l}$ loop in the calibration spot, and subsequently, CHCA ( $\alpha$-cyano-4-hydroxycinnamic acid) VITEK MS matrix solution was added [28]. After drying, the matrix/microorganism VITEK MS-DS slide was transferred to the instrument. The run was completed in 5 minutes [29].

FilmArray platform (BioFire technology, salt lake city, UT, USA)

The samples were directly taken from positive blood culture bottles. The FilmArray Blood Culture Identification (BCID) pouch was inserted into the loading station, following the color-coded guidelines. The hydration injection vials were injected into the FilmArray BCID panel pouches. The whole buffer was transferred into the sample buffer injection vial. Then, $0.2 \mathrm{ml}$ of the sample solution was transferred and mixed with the sample buffer. The sample/ buffer mix was injected into the pouch by a color-coded syringe [30]. The correct volume of the hydration solution and the sample was drawn due to the vacuum present in the pouch. Finally, the instrument lid was opened and the pouch was loaded according to the color guidelines. It took approximately 1 hour to finish the run. The software collected and analyzed the data from the patient samples along with the two internal QC reactions during the run. If one or two controls failed, the result was recorded as "invalid". A positive result indicated the detection of bacterial genus/species or resistance genes from a patient sample [31].

\section{The verigene (Nanosphere, Inc., Northbrook, IL, USA)}

The sample was directly taken from a positive blood cultures bottle. $700 \mu \mathrm{l}$ of the blood culture broth was added to the sample well on the right side of the extraction tray [32]. The instrument automatically checked the reagent and started the run. The test took $2.5 \mathrm{~h}$ to be completed. Subsequently, the test cartridge was removed from the Verigene Processor (SP), and the reagent pack was removed from the substrate holder, leaving the substrate holder on its side for 30 - 60 seconds. Finally, the protective tape was withdrawn from the pack of the substrate holder, and it was then subsequently inserted into the reader, which in turn provided the results after 5 minutes [13].

\section{Statistical analysis}

Data were analyzed using Statistical Package for Social Sciences (SPSS) software, version 19. The sensitivity, accuracy, Positive Predictive Value (PPV) for MALDI-TOF, Nanosphere, BioFire assays were calculated by comparing with the results obtained using the VITEK 2 identification method. Sensitivity was true-positive/(true positive + false- negatives) $\times 100$. Positive Predictive Value was
True Positive/(True Positive + False Positive). Accuracy was (True Positive + True Negative) $/($ True Positive + True Negative + False Positive + False Negative)

\section{Results}

In the first set of experiments, MALDI-TOF was used for the identification of Gram-positive bacteria, and the data was compared to the VITEK 2 method. Out of a total of 443 Gram-positive bacteria identified by VITEK 2, the MALDI-TOF method could identify 437. Therefore, the sensitivity of the MALDI-TOF for the identification of Gram-positive bacteria was 98.7\%, while PPV and accuracy were $99 \%$ and $98 \%$, respectively (Table 1 ).

\begin{tabular}{|c|c|c|c|c|c|}
\hline \multirow[b]{2}{*}{ Organism } & \multirow[b]{2}{*}{ VITEK 2} & \multicolumn{4}{|c|}{$\begin{array}{l}\text { No. of isolates identified by } \\
\text { MALDI-TOF }\end{array}$} \\
\hline & & $\begin{array}{c}\text { Cor- } \\
\text { rectly } \\
\text { identi- } \\
\text { fied }\end{array}$ & Not & $\begin{array}{c}\text { Identi- } \\
\text { fied as a } \\
\text { different } \\
\text { organism }\end{array}$ & $\begin{array}{l}\text { Not in } \\
\text { panel }\end{array}$ \\
\hline $\begin{array}{l}\text { Enterococcus } \\
\text { faecalis }\end{array}$ & 24 & 24 & 0.0 & 0.0 & 0.0 \\
\hline $\begin{array}{l}\text { Enterococcus } \\
\text { faecium }\end{array}$ & 14 & 14 & 0.0 & 0.0 & 0.0 \\
\hline $\begin{array}{l}\text { Leuconostoc } \\
\text { mesenteroides }\end{array}$ & 2 & 0.0 & 1 & $1^{*}$ & 0.0 \\
\hline $\begin{array}{l}\text { Staphylococcus } \\
\text { aureus }\end{array}$ & 111 & 111 & 0.0 & 0.0 & 0.0 \\
\hline $\begin{array}{l}\text { Staphylococcus } \\
\text { capitis }\end{array}$ & 27 & 27 & 0.0 & 0.0 & 0.0 \\
\hline $\begin{array}{l}\text { Staphylococcus } \\
\text { epidermidis }\end{array}$ & 133 & 133 & 0.0 & 0.0 & 0.0 \\
\hline $\begin{array}{l}\text { Staphylococcus } \\
\text { haemolyticus }\end{array}$ & 25 & 25 & 0.0 & 0.0 & 0.0 \\
\hline $\begin{array}{l}\text { Staphylococcus } \\
\text { hominis }\end{array}$ & 54 & 52 & 2 & 0.0 & 0.0 \\
\hline $\begin{array}{l}\text { Staphylococcus } \\
\text { intermedius }\end{array}$ & 1 & 1 & 0.0 & 0.0 & 0.0 \\
\hline $\begin{array}{l}\text { Staphylococcus } \\
\text { lugdunensis }\end{array}$ & 1 & 1 & 0.0 & 0.0 & 0.0 \\
\hline $\begin{array}{l}\text { Staphylococcus } \\
\text { saprophyticus }\end{array}$ & 1 & 1 & 0.0 & 0.0 & 0.0 \\
\hline $\begin{array}{l}\text { Staphylococcus } \\
\text { warneri }\end{array}$ & 2 & 2 & 0.0 & 0.0 & 0.0 \\
\hline $\begin{array}{l}\text { Streptococcus } \\
\text { agalactiae }\end{array}$ & 8 & 8 & 0.0 & 0.0 & 0.0 \\
\hline
\end{tabular}




\begin{tabular}{|l|c|c|c|c|c|}
\hline $\begin{array}{l}\text { Streptococcus } \\
\text { pneumoniae }\end{array}$ & 13 & 13 & 0.0 & 0.0 & 0.0 \\
\hline $\begin{array}{l}\text { Streptococcus } \\
\text { pyogenes }\end{array}$ & 8 & 7 & 1 & 0.0 & 0.0 \\
\hline $\begin{array}{l}\text { Streptococcus } \\
\text { viridans spp. }\end{array}$ & 11 & 11 & 0.0 & 0.0 & 0.0 \\
\hline $\begin{array}{l}\text { Streptococcus } \\
\text { anginosus }\end{array}$ & 3 & 3 & 0.0 & 0.0 & 0.0 \\
\hline $\begin{array}{l}\text { Streptococcus } \\
\text { mitis }\end{array}$ & 1 & 1 & 0.0 & 0.0 & 0.0 \\
\hline $\begin{array}{l}\text { Streptococcus } \\
\text { salivarius }\end{array}$ & 3 & 3 & 0.0 & 0.0 & 0.0 \\
\hline $\begin{array}{l}\text { Sporosarsina } \\
\text { pasteurii }\end{array}$ & 1 & 0.0 & 0.0 & 0.0 & 1 \\
\hline Total & 443 & 437 & $4(0.9 \%)$ & $1(0.2 \%)$ & $\begin{array}{c}1 \\
(0.2 \%)\end{array}$ \\
\hline
\end{tabular}

\begin{tabular}{|c|c|c|c|c|}
\hline $\begin{array}{l}\text { Burkholderia } \\
\text { серасia }\end{array}$ & 4 & 4 & 0.0 & 0.0 \\
\hline Citrobacter freundii & 4 & 4 & 0.0 & 0.0 \\
\hline $\begin{array}{l}\text { Enterobacter } \\
\text { aerogenes }\end{array}$ & 2 & 2 & 0.0 & 0.0 \\
\hline $\begin{array}{l}\text { Enterobacter } \\
\text { Cloacae }\end{array}$ & 23 & 22 & 1 & 0.0 \\
\hline Escherichia coli & 97 & 97 & 0.0 & 0.0 \\
\hline $\begin{array}{l}\text { Haemophilus } \\
\text { influenzae }\end{array}$ & 3 & 3 & 0.0 & 0.0 \\
\hline Klebsiella oxytoca & 1 & 1 & 0.0 & 0.0 \\
\hline $\begin{array}{l}\text { Klebsiella } \\
\text { pneumoniae }\end{array}$ & 97 & 96 & 1 & 0.0 \\
\hline $\begin{array}{l}\text { Morganella } \\
\text { morganii }\end{array}$ & 3 & 3 & 0.0 & 0.0 \\
\hline Pantoea & 1 & 1 & 0.0 & 0.0 \\
\hline $\begin{array}{l}\text { Pasteurella } \\
\text { multocida }\end{array}$ & 1 & 1 & 0.0 & 0.0 \\
\hline Proteus mirabilis & 15 & 15 & 0.0 & 0.0 \\
\hline Providencia rettgeri & 1 & 1 & 0.0 & 0.0 \\
\hline Providencia stuartii & 4 & 4 & 0.0 & 0.0 \\
\hline $\begin{array}{l}\text { Pseudomonas } \\
\text { aeruginosa }\end{array}$ & 41 & 41 & 0.0 & 0.0 \\
\hline Salmonella & 17 & 17 & 0.0 & 0.0 \\
\hline $\begin{array}{l}\text { Serratia } \\
\text { marcescens }\end{array}$ & 10 & 10 & 0.0 & 0.0 \\
\hline $\begin{array}{l}\text { Sphingomonas } \\
\text { paucimobilis }\end{array}$ & 5 & 5 & 0.0 & 0.0 \\
\hline $\begin{array}{l}\text { Stenotrophomonas } \\
\text { maltophilia }\end{array}$ & 12 & 12 & 0.0 & 0.0 \\
\hline Vibrio cholerae & 1 & 1 & 0.0 & 0.0 \\
\hline Delftia acidovorans & 1 & 0.0 & 0.0 & 1 \\
\hline Prevotella oralis & 1 & 0.0 & 0.0 & 1 \\
\hline Total & $\begin{array}{c}407 \\
(100 \%)\end{array}$ & $403(99 \%)$ & $\begin{array}{c}2 \\
(0.5 \%)\end{array}$ & $2(0.5 \%)$ \\
\hline
\end{tabular}

Similarly, we attempted to analyze the performance of MALDITOF as a tool to identify Gram-negative bacteria. We observed that MALDI-TOF was able to detect $403 \mathrm{Gram}$-negative bacteria out of the 407 Gram-negative bacteria identified by VITEK 2 (Table 2). Overall, the sensitivity of MALDI-TOF for the identification of Gramnegative organisms was $99 \%$, while PPV and accuracy were $100 \%$ and $99 \%$, respectively (Table 2).

\begin{tabular}{|l|c|c|c|c|}
\hline & & \multicolumn{3}{|c|}{$\begin{array}{c}\text { No. of isolates identified by } \\
\text { MALDI-TOF }\end{array}$} \\
\cline { 3 - 5 } Organism & VITEK 2 & $\begin{array}{c}\text { Correctly iden- Not de- } \\
\text { tified }\end{array}$ & $\begin{array}{c}\text { Not in } \\
\text { tected } \\
\text { panel }\end{array}$ \\
\hline $\begin{array}{l}\text { Achromobacter } \\
\text { denitrificans }\end{array}$ & 1 & 1 & 0.0 & 0.0 \\
\hline $\begin{array}{l}\text { Acinetobacter } \\
\text { baumannii }\end{array}$ & 55 & 55 & 0.0 & 0.0 \\
\hline $\begin{array}{l}\text { Aeromonas } \\
\text { hydrophil }\end{array}$ & 1 & 1 & 0.0 & 0.0 \\
\hline $\begin{array}{l}\text { Aeromonas sobria } \\
\text { Bacteroides fragilis }\end{array}$ & 1 & 1 & 0.0 & 0.0 \\
\hline $\begin{array}{l}\text { Bacteroides } \\
\text { uniform }\end{array}$ & 1 & 1 & 0.0 & 0.0 \\
\hline $\begin{array}{l}\text { Brevundimonas } \\
\text { diminuta }\end{array}$ & 1 & 1 & 0.0 & 0.0 \\
\hline
\end{tabular}

Table 2: Comparison between MALDI-TOF and VITEK 2 for the identification of Gram-negative organisms.

Next, Nanosphere was used for the identification of Gram-negative bacteria. Nanosphere could only identify 343 Gram-negative bacteria out of a total of 407 Gram-negative bacteria identified by VITEK 2 (Table 3). The sensitivity of the Nanosphere for the iden- 
tification of Gram-negative organisms was $84.3 \%$, while PPV and accuracy were $99 \%$ and $83 \%$, respectively. It is also important to note that a high percentage $(15.2 \%)$ of bacteria were not included in the panel of detection by Nanosphere (Table 3).

\begin{tabular}{|l|c|c|c|c|}
\hline \multirow{2}{*}{ Organism } & \multirow{2}{*}{ VITEK 2 } & \multicolumn{3}{|c|}{$\begin{array}{r}\text { No. of isolates identified by } \\
\text { Nanosphere }\end{array}$} \\
\cline { 3 - 5 } & & $\begin{array}{c}\text { Not } \\
\text { identified } \\
\text { detected }\end{array}$ & $\begin{array}{c}\text { Not in } \\
\text { panel }\end{array}$ \\
\hline Acinetobacter spp. & 55 & 55 & 0.0 & 0.0 \\
\hline Citrobacter spp. & 4 & 4 & 0.0 & 0.0 \\
\hline Enterobacter spp. & 25 & 25 & 0.0 & 0.0 \\
\hline Escherichia coli & 97 & 97 & 0.0 & 0.0 \\
\hline Klebsiella oxytoca & 1 & 1 & 0.0 & 0.0 \\
\hline $\begin{array}{l}\text { Klebsiella } \\
\text { pneumoniae }\end{array}$ & 97 & 96 & 1 & 0.0 \\
\hline Proteus spp. & 15 & 15 & 0.0 & 0.0 \\
\hline $\begin{array}{l}\text { Pseudomonas } \\
\text { aeruginosa }\end{array}$ & 41 & 41 & 0.0 & 0.0 \\
\hline $\begin{array}{l}\text { Serratia } \\
\text { marcescens }\end{array}$ & 10 & 9 & 1 & 0.0 \\
\hline * Other & 60 & 0.0 & 0.0 & 60 \\
\hline Total & & 343 & $2(0.5 \%)$ & 6 \\
\hline
\end{tabular}

Table 3: Comparison between Nanosphere and VITEK 2 for the identification of Gram-negative organisms.

*Other includes one Achromobacter denitrificans, one Aeromonas hydrophil, one Aeromonas sobria, three Bacteroides fragilis, one Bacteroides uniform, one Brevundimonas diminuta, four Burkholderia cepacia, one Delftia acidovorans, three Haemophilus influenzae, one Pasteurella multocida, one Prevotella oralis, one Providencia rettgeri, four Providencia stuartii, three Morganella morganii, seventeen Salmonella, five Sphingomonas paucimobilis, and fourteen Stenotrophomonas maltophilia.

BioFire was the next rapid technique that was analyzed for the detection of Gram-positive bacteria. In this study, BioFire could identify 435 Gram-positive bacteria as compared to 443 Gram-positive bacteria identified by the VITEK 2 (Table 4). The sensitivity of BioFire for the identification of Gram-positive bacteria was $98.2 \%$, while PPV and accuracy were 99\% and 98.2\%, respectively (Table 4).

\begin{tabular}{|l|c|c|c|c|}
\hline \multirow{2}{*}{ Organism } & \multirow{2}{*}{ VITEK 2 } & \multicolumn{3}{|c|}{$\begin{array}{c}\text { No. of isolates identified by } \\
\text { BioFire }\end{array}$} \\
\cline { 3 - 5 } & & $\begin{array}{c}\text { Correctly } \\
\text { identified }\end{array}$ & $\begin{array}{c}\text { Not } \\
\text { detected }\end{array}$ & $\begin{array}{c}\text { Not in } \\
\text { panel }\end{array}$ \\
\hline $\begin{array}{l}\text { Coagulase-negative } \\
\text { Staphylococci (CoNS) }\end{array}$ & 244 & 241 & 3 & 0.0 \\
\hline Enterococcus & 38 & 38 & 0.0 & 0.0 \\
\hline Staphylococcus aureus & 111 & 110 & 1 & 0.0 \\
\hline Streptococcus & 8 & 8 & 0.0 & 0.0 \\
\hline agalactiae & 13 & 12 & 1 & 0.0 \\
\hline $\begin{array}{l}\text { Streptococcus } \\
\text { pneumoniae }\end{array}$ & 8 & 8 & 0.0 & 0.0 \\
\hline Streptococcus pyogenes & 18 & 18 & 0.0 & 0.0 \\
\hline Streptococcus spp. & 1 & 0.0 & 0.0 & 1 \\
\hline $\begin{array}{l}\text { Leuconostoc } \\
\text { mesenteroides }\end{array}$ & $100 \%)$ & $(98.2 \%)$ & $5(1.1 \%)$ & $3(0.7 \%)$ \\
\hline Sporosarsina pasteurii & 1 & 0.0 & 0.0 & 2 \\
\hline Total & 183 & & & \\
\hline
\end{tabular}

Table 4: Comparison between BioFire and VITEK 2 for the identification of Gram-positive organisms.

Finally, the performance of the BioFire was evaluated for the detection of different isolates of yeast. In our study, BioFire was able to identify 59 yeast isolates as compared to a total of 66 yeast strains identified by VITEK 2 (Table 5). The final results showed that the sensitivity of BioFire to identify yeast strains was $89.4 \%$, while PPV and accuracy were $100 \%$ and $89 \%$, respectively (Table 5).

\section{Discussion}

Identifying bacteria from positive blood cultures by a conventional method usually takes several days [33]. VITEK 2 identifies bacteria, yeast, and determines the antibiotic susceptibility with accuracy, providing precise results; the results for Gram-positive bacteria take approximately 19 hours, 7 - 9 hours for Gram-negative bacteria and 24 - 48 hours for yeast [25,34]. 


\begin{tabular}{|l|c|c|c|c|}
\hline \multirow{2}{*}{ Organism } & \multirow{2}{*}{ VITEK 2 } & \multicolumn{3}{|c|}{$\begin{array}{r}\text { No. of isolates identified by } \\
\text { BioFire }\end{array}$} \\
\cline { 3 - 5 } & & $\begin{array}{c}\text { Correctly } \\
\text { identified }\end{array}$ & $\begin{array}{c}\text { Not } \\
\text { detected }\end{array}$ & $\begin{array}{c}\text { Not in } \\
\text { Panel }\end{array}$ \\
\hline Candida albicans & 23 & 23 & 0.0 & 0.0 \\
\hline Candida glabrata & 4 & 4 & 0.0 & 0.0 \\
\hline Candida parapsilosis & 15 & 15 & 0.0 & 0.0 \\
\hline Candida topicalis & 18 & 17 & 1 & 0.0 \\
\hline Candida dubliniensis & 1 & 0.0 & 0.0 & 1 \\
\hline Cryptococcus neoformans & 4 & 0.0 & 0.0 & 4 \\
\hline Trichosporon asahii & 1 & 0.0 & 0.0 & 1 \\
\hline Total & 66 & 59 & $1(1.5 \%)$ & $6(9.1 \%)$ \\
\hline
\end{tabular}

Table 5: Comparison between BioFire and VITEK 2 for the identification of yeasts.

The non conventional available rapid technologies such as MALDI-TOF, BioFire, and Nanosphere have contributed to accelerate the process of identifying microorganisms [35-39]. Moreover, BioFire and Nanosphere can also detect some of the antimicrobial resistance genes quickly, and the results are highly accurate [40]. This study aimed to compare the non conventional rapid technologies for bacterial identification versus the conventional methods. Additionally, we also ascertained the advantages and disadvantages of using these technologies for BSI detection.

In our study, 437 (98.7\%) bacteria were identified out of a total of 443 Gram-positive bacteria using MALDI-TOF. The sensitivity, PPV, and accuracy of MALDI-TOF for detecting Gram-positive bacteria at the species level were $98.7 \%, 99 \%$, and $98 \%$, respectively (Table 1). These results agreed with the findings of Eigner., et al. [41], who reported that the accuracy of MALDI-TOF for the identification of Gram-positive bacteria was 95.2\%. Also, Rychert., et al. [42] reported that the sensitivity and accuracy of MALDI-TOF in Gram-positive bacterial identification were $92.8 \%$ and $95 \%$, respectively. Our study found five positive blood cultures with Grampositive strains that MALDI-TOF could not detect; Sporosarsina pasteurii was not in the panel while one Leuconostoc mesenteroides, two $S$. hominis, and one $S$. pyogenes were possibly not detected due to technical errors (Table 1).
It was surprising that the other isolate of Leuconostoc mesenteroides was identified by MALDI-TOF as Wasilla confusa; both $L$. mesenteroides and $W$. confusa are included in the Leuconostocaceae family due to their similarity (Table 1 ). Similar results were also observed by Lee., et al. [43], who stated that the isolates of Weissella species recovered from the blood cultures could be misidentified as Leuconostoc species by traditional or commercial phenotypic identification methods.

MALDI-TOF identified eleven samples of Streptococcus viridans at the genus level and seven samples at the species level along with three isolates of $S$. anginosus, one $S$. mitis, and three $S$. salivarius (Table 1). These results are in contrast with Kaleta., et al. [44], who concluded that MALDI-TOF MS could be easily used in routine identification of bacteria in a medical microbiological laboratory except for pneumococci and viridans streptococci; 12 out 21 (57.1\%) viridans streptococci were erroneously identified as $S$. pneumoniae.

In the present study, MALDI-TOF correctly identified 403 (99\%) bacteria out of 407 Gram-negative bacteria at the species and genus levels. The sensitivity, PPV, and accuracy of this method were $99 \%, 100 \%$, and $99 \%$, respectively. There was no misidentification in the results (Table 2). These results were consistent with those of Richter., et al. [45], who reported that MALDI-TOF correctly identified Gram-negative strains at the genus level, and $83.8 \%$ of them were identified to the species level.

In our study, there were four Gram-negative bacteria that were not detected by MALDI-TOF; two of them were not in the panel (Delftia acidovorans and Prevotella oralis). The other two undetected bacteria were K. pneumoniae and Enterobacter cloaca, which may be attributed to technical errors.

In the current study, we observed that 343 (84.3\%) out of 405 Gram-negative bacteria were correctly identified by Nanosphere (BC-GN). The sensitivity, PPV, and accuracy levels of the Nanosphere in detecting Gram-negative bacteria were 84.3\%, 99\%, and $83 \%$, respectively (Table 3 ). These sensitivity values obtained in our study were lower than the results presented in a study by Bork., et al. [46], where the Nanosphere (BC-GN) correctly identified Gram-negative bacteria with a sensitivity of $95.6 \%$.

This difference may be due to the presence of 62 bacterial isolates in our study that are not included in the panel of detection by Nanosphere, and the details of those isolates are as follows: one Achromobacter denitrificans, one Aeromonas hydrophil, 
one Aeromonas sobria, one Brevundimonas diminuta, one Delftia acidovorans, one Prevotella oralis, one Pasteurella multocida, one Providencia rettgeri, four Providencia stuartii, three Bacteroides fragilis, one Bacteroides uniform, four Burkholderia cepacia, three Haemophilus influenzae, three Morganella morganii, seventeen Salmonella, five Sphingomonas paucimobilis and fourteen Stenotrophomonas maltophilia (Table 3).

It is also worth noting that Nanosphere did not show any misidentification result in our study. These results are better in comparison to the study performed by Ledeboer and his co-investigators [32], who had reported one false-positive result where Raoultella species was misidentified as K. oxytoca. This misidentification was possibly due to the phenotypical and genotypical similarity between $K$. oxytoca and Raoultella.

There were interesting findings when the samples were analyzed from two different blood culture bottles. The first blood culture bottle contained both Serratia marcescens and Pseudomonas aeruginosa, as detected by VITEK 2, whereas the Nanosphere could only identify $S$. marcescens. The second blood culture bottle contained both $K$. pneumoniae and E. cloacae, which were both detected by VITEK 2, however, the Nanosphere could only detect $E$. cloacae strain. These findings agree entirely with the Sullivan., et al. study [12], where they reported that the Verigene BC-GN microarray might be less sensitive in detecting mixed Gram-negative bacteremia.

Ledeboerand and his co-investigators [32] showed that the Nanosphere BC-GN assay accurately identified at least one organism existing in $95 \%$ of the polymicrobial cultures tested; nevertheless, the correct identification of all the microorganisms present in the culture was achieved only for $54 \%$ of these specimens. Also, Dodémontand his colleagues [15] stated that the identification of Gram-negative organisms and resistance genes was more accurate in a monomicrobial (99\%) culture than in a polymicrobial culture $(83.3 \%)$.

On the other hand, there was a significant finding in one of the positive blood culture bottles, where Nanosphere BC-GN identified four distinct bacteria (K. pneumoniae, E. cloacae, E. coli, and $S$. marcescens). While only $K$. pneumoniae was isolated from the subculture and was identified by VITEK 2 . This result corresponds with the findings of Sullivan and his co-investigators in 2014 [12] who found that, in a single blood culture, Nanosphere BC-GN identified Citrobacter spp. and K. pneumoniae, while only K. pneumoni- ae could be isolated from the subculture. The authors hypothesized that these findings might be due to false-positive Nanosphere BCGN results or detection of the DNA of dead bacilli.

In our study, we found that BioFire correctly identified 435 (98.2\%) Gram-positive bacteria out of a total of 443 isolates. The sensitivity, PPV, and accuracy levels of BioFire were 98.2\%, 99\%, and $98.2 \%$, respectively (Table 4). These results agree with Bhatti., et al. [47], who found that the sensitivity of Gram-positive bacterial identification by Biofire was $94 \%$. Meanwhile, Fhooblall and his colleagues [48] reported that the sensitivity of BioFire was $93.8 \%$ for monomicrobial Gram-positive specimens. Additionally, similar results were also reported in a study by Fiori., et al. [30], which stated that the sensitivity and PPV of BioFire for Gram-positive bacterial identification were $97.7 \%$ and $100 \%$, respectively.

Furthermore, in our study BioFire was unable to detect Grampositive bacteria isolated from five positive blood cultures. It was noticed that two of them were polymicrobial samples, one sample contained both $S$. epidermidis and C. parapsilosis, and BioFire couldn't detect $S$. epidermidis when it was present with $C$. parapsilosis. Also, Biofire couldn't detect $S$. aureus when it was present with E. fecalis. On the other hand, the third, fourth, and fifth samples, which were monomicrobial, the Biofire could not detect two samples containing $S$. hominis and one sample containing $S$. pneumoniae. Finally, three organisms that were not in the BioFire panel were two L. mesenteroides and one S. pasteurii_(Table 4).

These findings were similar to the findings of Zheng and his coinvestigators [7], who reported that there were 4 different bacteria that BioFire could not identify. Two of them were Staphylococcus hominis, present in a mixed culture with Gram-positive coccus in clusters. The other two were Enterococcus faecium, present in a mixed culture with Gram-negative rods and identified correctly, and a viridians streptococcus, mixed with Staphylococcus capitis.

All the results mentioned above agree with the study of Altun and co-investigators [11], who found that BioFire could detect only $71 \%$ of the organisms isolated from polymicrobial growth bottles and $91.6 \%$ of the microorganisms from monomicrobial growth bottles.

In the present study, 59 yeast strains out of a total of 66 (89.4\%) were correctly identified by BioFire. The sensitivity of BioFire was $89.4 \%$, PPV was $100 \%$, and the accuracy was $89 \%$ (Table 5). This result was in agreement with Fiori., et al. [30], who reported a sen- 
sitivity of $98.6 \%$ for the identification of yeast by BioFire in monomicrobial blood culture bottles. On the other hand, Fiori and his colleagues [30] reported that the FilmArray BCID panel correctly identified $94.0 \%$ of the Gram-positive bacteria and $100 \%$ of the yeast strains recovered from polymicrobial cultures.

Due to limited yeast strains in the BioFire panel, six fungi were not identified from positive blood cultures: Candida dubliniensis (1 isolate), Cryptococcus neoformans (4 isolates), and Trichosporonasahii (1 isolate), thereby reducing the overall sensitivity of the BioFire. One of the yeast isolate included in the BioFire panel and not detected was Candida tropicalis, which may be due to a technical error (Table 5).

There were five polymicrobial positive blood cultures for yeast as detected by BioFire. However, VITEK 2 failed to identify those five positive cultures in the first attempt. Therefore, the test was repeated via VITEK 2 , and they were correctly identified. This suggests that having pure isolated yeast colonies on the Sabouraud dextrose agar plate plays an important role in the identification of the microorganisms as there is a similarity in the morphology of the yeast colonies. In this study, these discrepant results were not included in our data calculation.

Fhooblalland and co-investigators [48] stated that the number of organisms detected by BioFire, particularly in blood cultures, was more than what the reference method VITEK 2 could identify. The higher number of organisms identified by BioFire may have been due to the detection of the nucleic acid of non-viable organisms present in the blood culture.

BioFire and Nanosphere have a limited number of targets included in their panels. One of the limitations of the Nanosphere assays is the need for a Gram stain before an appropriate panel is chosen $[15,47,48]$. However, one solution to this problem is that, at a specific time, one sample can be run in multiple instruments in parallel. Until now, the extensive use of the BioFire BCID panel in routine clinical laboratory practice has been discouraged due to the high cost of reagents/consumables [11]. MALDI-TOF can rapidly identify many organisms up to species level, however, it does not provide information about the antimicrobial-susceptibility [4].

Therefore, rapid microbiological investigations that identify the causative agent and AST are vital to choose the correct antimicrobial therapy and avoid inappropriate treatment. Additionally, a reduction in the spectrum of antimicrobial agents used will help limit the emergence of resistant strains. In addition, limited use of broad-spectrum antibiotics or combined therapy will also reduce the toxicity and negative impact on the beneficial bacteria.

\section{Conclusion}

Our assessment of the MALDI-TOF, BioFire, and Nanosphere systems on the positive blood cultures demonstrated realistic accuracy and practical benefits for the rapid identification of bacteria in monomicrobial and polymicrobial blood cultures. Moreover, these technologies can markedly reduce the turnaround time in the microbiology laboratories of many hospitals. Thus, if utilized properly, these techniques would improve the choice of the most appropriate antimicrobials administered to the patients and significantly improve patient management. However, these systems cannot replace the conventional culture method. The growth of the bacterial colonies is necessary to identify microorganisms that are not included in the panels and complete the susceptibility testing.

\section{Acknowledgments}

The authors would like to thank Miss. Rajaa Al-Ahmadi for her assistance in performing statistical analyses.

\section{Bibliography}

1. Son JS., et al. "Bloodstream infections and clinical significance of healthcare-associated bacteremia: a multicenter surveillance study in Korean hospitals". Journal of Korean Medical Science 25.7 (2010): 992-998.

2. Prabhash K., et al. "Blood stream infections in cancer patients: a single center experience of isolates and sensitivity pattern". Indian Journal of Cancer 47.2 (2010): 184-188.

3. Hassan M., et al. "Cost of hospital-acquired infection". Hospital Topics 88.3 (2010): 82-89.

4. Samuel LP., et al. "Evaluation of a microarray-based assay for rapid identification of Gram-positive organisms and resistance markers in positive blood cultures". Journal of Clinical Microbiology 51.4 (2013): 1188-1192.

5. Ecker DJ., et al. "New technology for rapid molecular diagnosis of bloodstream infections". Expert Review of Molecular Diagnostics 10.4 (2010): 399-415.

6. Carrara L., et al. "Molecular diagnosis of bloodstream infections with a new dual-priming oligonucleotide-based multiplex PCR assay". Journal of Medical Microbiology 62.11 (2013): 1673-1679. 
7. Zheng X., et al. "Rapid identification of pathogens from pediatric blood cultures by use of the FilmArray blood culture identification panel". Journal of Clinical Microbiology 52.12 (2014): 4368-4371.

8. Ward C., et al. "Performance evaluation of the Verigene ${ }^{\circledR}$ (Nanosphere) and FilmArray® (BioFire $®$ ) molecular assays for identification of causative organisms in bacterial bloodstream infections". European Journal of Clinical Microbiology and Infectious Diseases 34.3 (2015): 487-496.

9. Schmidt V., et al. "Rapid identification of bacteria in positive blood culture by matrix-assisted laser desorption ionization time-of-flight mass spectrometry". European Journal of Clinical Microbiology \& Infectious Diseases 31.3 (2012): 311-317.

10. Salimnia H., et al. "Evaluation of the FilmArray Blood Culture Identification Panel: Results of a Multicenter Controlled Trial". Journal of Clinical Microbiology 54.3 (2016): 687-698.

11. Altun 0., et al. "Clinical evaluation of the FilmArray blood culture identification panel in identification of bacteria and yeasts from positive blood culture bottles". Journal of Clinical Microbiology 51.12 (2013): 4130-4136.

12. Sullivan KV., et al. "Pediatric multicenter evaluation of the Verigene gram-negative blood culture test for rapid detection of inpatient bacteremia involving gram-negative organisms, extended-spectrum beta-lactamases, and carbapenemases". Journal of Clinical Microbiology 52.7 (2014): 2416-2421.

13. Beal SG., et al. "Evaluation of the nanosphere verigene grampositive blood culture assay with the VersaTREK blood culture system and assessment of possible impact on selected patients". Journal of Clinical Microbiology 51.12 (2013): 39883992.

14. Beal SG., et al. "Antibiotic utilization improvement with the Nanosphere Verigene Gram-Positive Blood Culture assay". Baylor University Medical Center Proceedings 28.2 (2015): 139-143.

15. Dodemont M., et al. "Performance of the Verigene Gram-negative blood culture assay for rapid detection of bacteria and resistance determinants". Journal of Clinical Microbiology 52.8 (2014): 3085-3087.
16. Arvanitis M., et al. "Molecular and nonmolecular diagnostic methods for invasive fungal infections". Clinical Microbiology Reviews 27.3 (2014): 490-526.

17. Liesenfeld 0., et al. "Molecular diagnosis of sepsis: New aspects and recent developments". European Journal of Microbiology and Immunology 4.1 (2014): 1-25.

18. Lavergne R-A., et al. "An extraction method of positive blood cultures for direct identification of Candida species by Vitek MS matrix-assisted laser desorption ionization time of flight mass spectrometry". Medical Mycology 51.6 (2013): 652-656.

19. Afshari A., et al. "Bench-to-bedside review: Rapid molecular diagnostics for bloodstream infection - a new frontier?" Critical Care 16.3 (2012): 222.

20. Cherkaoui A., et al. "Comparison of two matrix-assisted laser desorption ionization-time of flight mass spectrometry methods with conventional phenotypic identification for routine identification of bacteria to the species level". Journal of Clinical Microbiology 48.4 (2010): 1169-1175.

21. Carbonnelle E., et al. "MALDI-TOF mass spectrometry tools for bacterial identification in clinical microbiology laboratory". Clinical Biochemistry 44.1 (2011): 104-109.

22. Zwadyk P Jr., et al. "Comparison of Difco ESP and Organon Teknika BacT/Alert continuous-monitoring blood culture systems". Journal of Clinical Microbiology 32.5 (1994): 12731279.

23. Kocoglu ME., et al. "Evaluation of negative results of BacT/ Alert 3D automated blood culture system". Journal of Microbiology 43.3 (2005): 257-259.

24. Livermore DM., et al. "Multicentre evaluation of the VITEK 2 Advanced Expert System for interpretive reading of antimicrobial resistance tests". Journal of Antimicrobial Chemotherapy 49.2 (2002): 289-300.

25. Wallet F., et al. "Performances of VITEK 2 colorimetric cards for identification of gram-positive and gram-negative bacteria”. Journal of Clinical Microbiology 43.9 (2005): 4402-4406.

26. Pincus DH. "Microbial identification using the bioMérieuxVitek® 2 system". In Encyclopedia of Rapid Microbiological Methods; Parenteral Drug Association: Bethesda, MD, USA, (2006): $1-32$. 
27. Hata DJ., et al. "Multicenter evaluation of the new VITEK 2 advanced colorimetric yeast identification card". Journal of Clinical Microbiology 45.4 (2007): 1087-1092.

28. Dubois D., et al. "Performances of the Vitek MS matrix-assisted laser desorption ionization-time of flight mass spectrometry system for rapid identification of bacteria in routine clinical microbiology". Journal of Clinical Microbiology 50.8 (2012): 2568-2576.

29. Patel R. "MALDI-TOF MS for the diagnosis of infectious diseases". Clinical Chemistry 61.1 (2015): 100-111.

30. Fiori B., et al. "Optimized Use of the MALDI BioTyper System and the FilmArray BCID Panel for Direct Identification of Microbial Pathogens from Positive Blood Cultures". Journal of Clinical Microbiology 54.3 (2016): 576-584.

31. Poritz MA., et al. "FilmArray, an automated nested multiplex PCR system for multi-pathogen detection: development and application to respiratory tract infection". PLOS ONE 6.10 (2011): e26047.

32. Ledeboer NA., et al. "Identification of Gram-Negative Bacteria and Genetic Resistance Determinants from Positive Blood Culture Broths by Use of the Verigene Gram-Negative Blood Culture Multiplex Microarray-Based Molecular Assay". Journal of Clinical Microbiology 53.8 (2015): 2460-2472.

33. Martinez RM., et al. "Evaluation of three rapid diagnostic methods for direct identification of microorganisms in positive blood cultures". Journal of Clinical Microbiology 52.7 (2014): 2521-2529.

34. Aubertine CL., et al. "Comparative study of the new colorimetric VITEK 2 yeast identification card versus the older fluorometric card and of CHROMagar Candida as a source medium with the new card". Journal of Clinical Microbiology 44.1 (2006): 227-228.

35. Huang AM., et al. "Impact of rapid organism identification via matrix-assisted laser desorption/ionization time-of-flight combined with antimicrobial stewardship team intervention in adult patients with bacteremia and candidemia". Clinical Infectious Diseases 57.9 (2013): 1237-1245.
36. Luethy PM., et al. "The Use of Matrix-Assisted Laser Desorption/Ionization Time-of-Flight Mass Spectrometry (MALDITOF MS) for the Identification of Pathogens Causing Sepsis". The Journal of Applied Laboratory Medicine 3.4 (2019): 675685.

37. Dai Y., et al. "Evaluation of a Rapid and Simplified Protocol for Direct Identification of Microorganisms From Positive Blood Cultures by Using Matrix Assisted Laser Desorption Ionization Time-of-Flight Mass Spectrometry (MALDI-TOF MS)". Frontiers in Cellular and Infection Microbiology 11 (2021): 632679.

38. Howard AK., et al. "Performance of Verigene Rapid Diagnostic Testing for Detection of Inpatient Pediatric Bacteremia". The Journal of Pediatric Pharmacology and Therapeutics 26.5 (2021): 472-477.

39. Tansarli GS., et al. "Diagnostic test accuracy of the BioFire ${ }^{\circledR}$ FilmArray® meningitis/encephalitis panel: a systematic review and meta-analysis". Clinical Microbiology and Infection 26.3 (2020): 281-290.

40. Goff DA., et al. "Using rapid diagnostic tests to optimize antimicrobial selection in antimicrobial stewardship programs". Pharmacotherapy 32.8 (2012): 677-687.

41. Eigner U., et al. "Performance of a matrix-assisted laser desorption ionization-time-of-flight mass spectrometry system for the identification of bacterial isolates in the clinical routine laboratory". Clinical Laboratory 55 (2009): 289-296.

42. Rychert J., et al. "Multicenter evaluation of the Vitek MS matrix-assisted laser desorption ionization-time of flight mass spectrometry system for identification of Gram-positive aerobic bacteria". Journal of Clinical Microbiology 51.7 (2013): 2225-2231.

43. Lee MR., et al. "Bacteraemia caused by Weissella confusa at a university hospital in Taiwan, 1997-2007". Clinical Microbiology and Infection 17.8 (2011): 1226-1231.

44. Kaleta EJ., et al. "Comparative analysis of PCR-electrospray ionization/mass spectrometry (MS) and MALDI-TOF/MS for the identification of bacteria and yeast from positive blood culture bottles". Clinical Chemistry 57.7 (2011): 1057-1067. 
45. Richter SS., et al. "Identification of Enterobacteriaceae by matrix-assisted laser desorption/ionization time-of-flight mass spectrometry using the VITEK MS system". European Journal of Clinical Microbiology and Infectious Diseases 32.12 (2013): 1571-1578.

46. Bork JT., et al. "Rapid testing using the Verigene Gram-negative blood culture nucleic acid test in combination with antimicrobial stewardship intervention against Gram-negative bacteremia". Antimicrobial Agents and Chemotherapy 59.3 (2015): 1588-1595.

47. Bhatti MM., et al. "Evaluation of FilmArray and Verigene systems for rapid identification of positive blood cultures". Journal of Clinical Microbiology 52.9 (2014): 3433-3436.

48. Fhooblall M., et al. "Evaluation of the BioFire® FilmArray® Blood Culture Identification Panel on positive blood cultures in a regional hospital laboratory in KwaZulu-Natal". African Journal of Laboratory Medicine 5.1 (2016): 411.

Volume 4 Issue 9 September 2021

(C) All rights are reserved by Dalia EL-Hossary., et al. 